\title{
Praxisführung für Grundversorger*: Vorbereitung auf den Einstieg in die eigene Praxis
}

Bei ausschliesslich männlichen Formulierungen ist das weibliche Geschlecht mitgemeint und umgekehrt
Das Kursmodul des Kollegiums für Hausarztmedizin wird in diesem Jahr zum dritten Mal angeboten. Es richtet sich an Kolleginnen und Kollegen, die 1-2 Jahre vor dem Einstieg in die eigene Praxis stehen und einen intensiven Gedanken- und Erfahrungsaustausch zu Themen wie zum Beispiel «Wie führe ich eine eigene Praxis?», «Wie organisiere ich meinen Betrieb am besten?», «Wie setze ich meine Ressourcen optimal ein?» suchen. Die Themen und Schwerpunkte werden dabei von den Teilnehmerinnen weitgehend selber festgelegt.

Das Weiterbildungsmodul Praxisführung ist berufsbegleitend über eine Dauer von 6 Monaten angelegt und berücksichtigt moderne Grundsätze der Erwachsenenbildung.

Alle Teilnehmenden analysieren in der Startphase ihre individuellen Bedürfnisse und Lücken mit Hilfe eines speziell für die hausärztliche Praxisführung entwickelten Instruments. Daraus ergibt sich ein individueller Lernplan, der in den Präsenzteilen und in der Selbstlernphase umgesetzt wird. Dieses «Self-Assessment» umfasst wichtige Fragen und Stichworte zu folgenden Themen:

- Selbstmanagement: Hier geht es um Zeitund Aufgabengestaltung, Umgang mit persönlichen und beruflichen Belastungen, Stressmanagement und Burn-out-Prophylaxe, Gleichgewicht zwischen Beruf und anderen Lebensbereichen, neue Arbeitszeit- und Praxismodelle, Massnahmen zur Erhaltung und Förderung der beruflichen Kompetenz.

- Der Bereich Praxis-Management umfasst die ganze Breite der unternehmerischen Aufgaben: Menschenführung (Personalselektion, -führung und -entwicklung; Teamarbeit; Kommunikation und Umgang mit Patienten); Demand-, Disease- und Case-Management; die Organisation des Praxisbetriebes (Betrieb, Finanzen, Informationsfluss, Dokumentation).
- Die Vernetzung zwischen Praxis und Umfeld wird immer wichtiger: Zusammenarbeit mit Kollegen, medizinischen und sozialen Diensten, Behörden, Versicherungen; Aufgaben für die öffentliche Gesundheit; berufsund allgemeinpolitisches Engagement inkl. dafür erforderlicher Kommunikationsfertigkeiten, Ethik, Qualitätssicherung und Praxisforschung, Umweltanliegen.

Im ersten Präsenzkurs werden die Grundlagen für eine erfolgreiche Selbst- und Gruppenlernphase gelegt und einige wesentliche Kerninhalte im Bereich Selbstmanagement und zu Praxisformen (Einzelpraxis, Gruppenpraxis) vermittelt (Dauer anderthalb Tage).

In der Selbst- und Gruppenlernphase arbeiten die Teilnehmenden, unterstützt durch die Kursleitung, über eine Dauer von 5 Monaten, um ihre individuellen Lernziele zu erreichen. Sie lernen selbständig und tauschen Informationen über E-Mail und über die Webplattform des Weiterbildungsmoduls aus. Literatur und Erfahrungsberichte sowie eigene Arbeiten können über die webbasierte Dokumentationsplattform allen Teilnehmenden zur Verfügung gestellt werden. Die Webplattform enthält auch weitere Kursunterlagen, Begleitmaterialien und Literatur, Links, Adresslisten und eine Datenbank mit Drittangeboten. Auf Wunsch besteht die Möglichkeit von Webmeetings, und die Kursleitung unterstützt auch Gruppentreffen.

Im zweiten Präsenzkurs von wiederum anderthalb Tagen präsentieren die Teilnehmerinnen und Teilnehmer ihre Erfahrungen und Arbeiten den anderen Kolleginnen und Kollegen des Kurses. Es sollen eine intensive Diskussion und ein intensiver Erfahrungsaustausch stattfinden. Bei Bedarf können externe Experten zugezogen werden. Kursleitung und Teilnehmende bauen laufend eine Wissensdatenbank und eine Linksammlung zu Fragen der Praxisführung auf. 


\section{Teilnahmebedingungen}

In Weiterbildung zur Hausärztin / zum Hausarzt (Allgemeinmedizin, Innere Medizin oder Pädiatrie) ca. 1-3 Jahre vor der Praxiseröffnung. Zugang zum Internet und gute E-Mail-Kommunikation. Kurssprache: Deutsch.

\section{Kursgebühr}

Fr. 800.-. Die Kosten für die Unterkunft und Verpflegung (ca. Fr. 200.-) an den Präsenzkursen sind durch die Teilnehmer direkt vor Ort zu bezahlen.

Ab sofort ist es möglich, im November oder im Mai in den Kurs einzusteigen. Die Präsenzkurse werden für die Teilnehmenden der beiden Kursmodule parallel durchgeführt.

\section{Termine Präsenzkurse}

24./25.8.2007: Anmeldeschluss 30.4.2007

18./19.1.2008: Anmeldeschluss 30.9.2007

22./23.8.2008: Anmeldeschluss 30.4.2008

(Zwischen Anmeldeschluss und Präsenzkurs läuft die Startphase mit dem Self-Assessment.)

\section{Informationen und Anmeldung}

Kollegium für Hausarztmedizin, Landhausweg 26, 3007 Bern, Tel. 03137006 70, E-Mail: khm@ hin.ch, www.kollegium.ch/pf (Informationen und Flyer zum Herunterladen). 